\title{
Anhang zur quantitativen Studie
}

Denisa Kykalová

10.1 Teilnehmerkreis der Online-Befragung - 140

10.2 Fragenkatalog der Online-Befragung - 144 


\subsection{Teilnehmerkreis der Online-Befragung}

TEILNEHMENDE NACH LAND DER

GESCHÄFTSTÄTIGKEIT DES

UNTERNEHMENS

\section{Schweiz}

Deutschland

Andere

Österreich

$82 \%$
$12 \%$
$5 \%$
$1 \%$

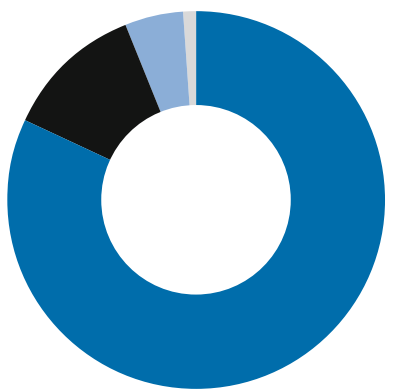

$\mathrm{N}=178$

- Abb. 10.1 Teilnehmende nach Land der Geschäftstätigkeit des Unternehmens

TEILNEHMENDE NACH GRÖSSE

DES UNTERNEHMENS

1-9 Mitarbeitende
10-49
$50-249$
250 und mehr

$9,6 \%$

$16,9 \%$

$12,9 \%$

250 und mehr

$60,7 \%$

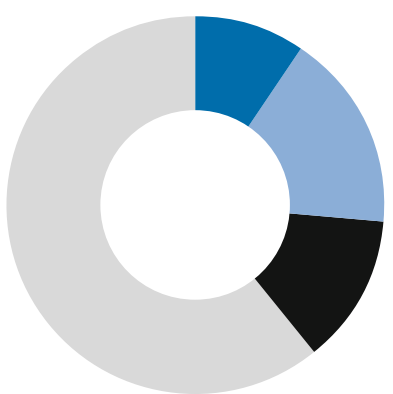

- Abb. 10.2 Teilnehmende nach Grösse des Unternehmens 


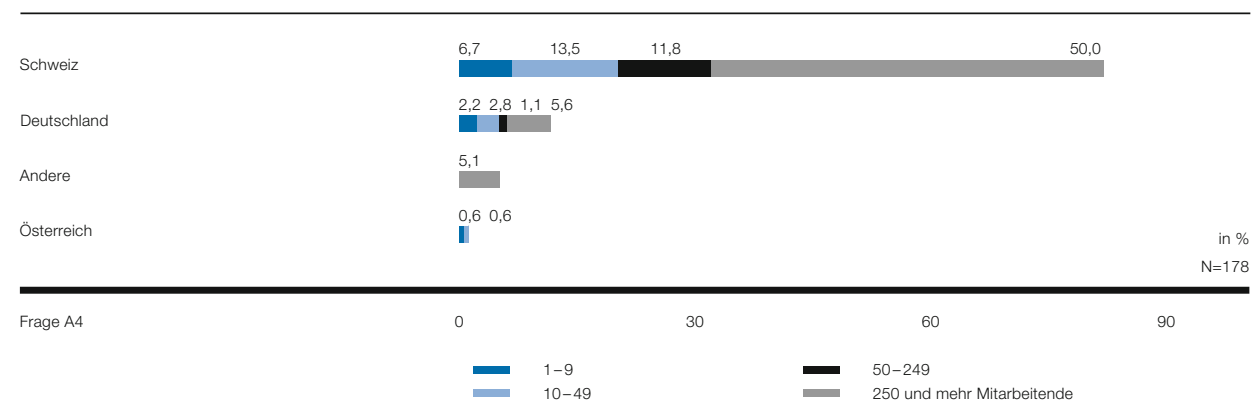

- Abb. 10.3 Teilnehmende nach Land und Unternehmensgrösse

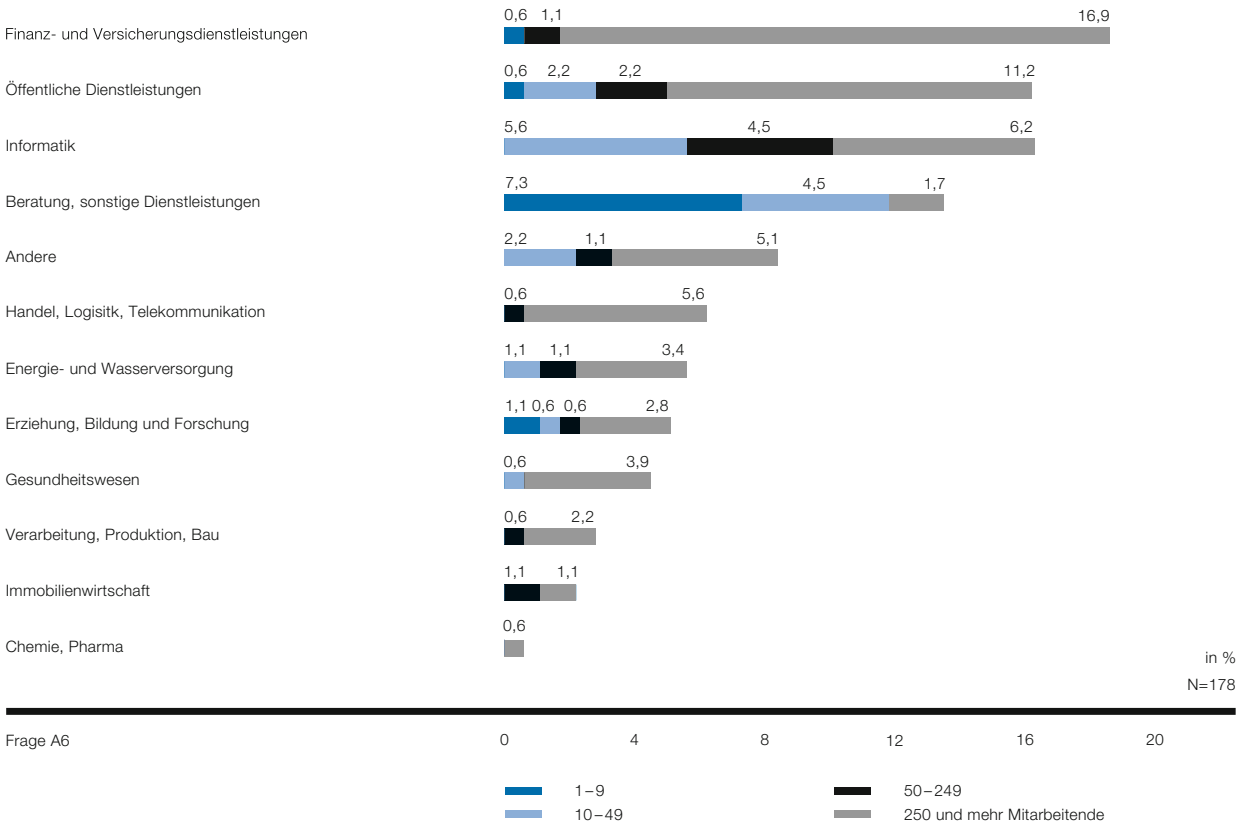

- Abb. 10.4 Teilnehmende nach Branchengruppe 


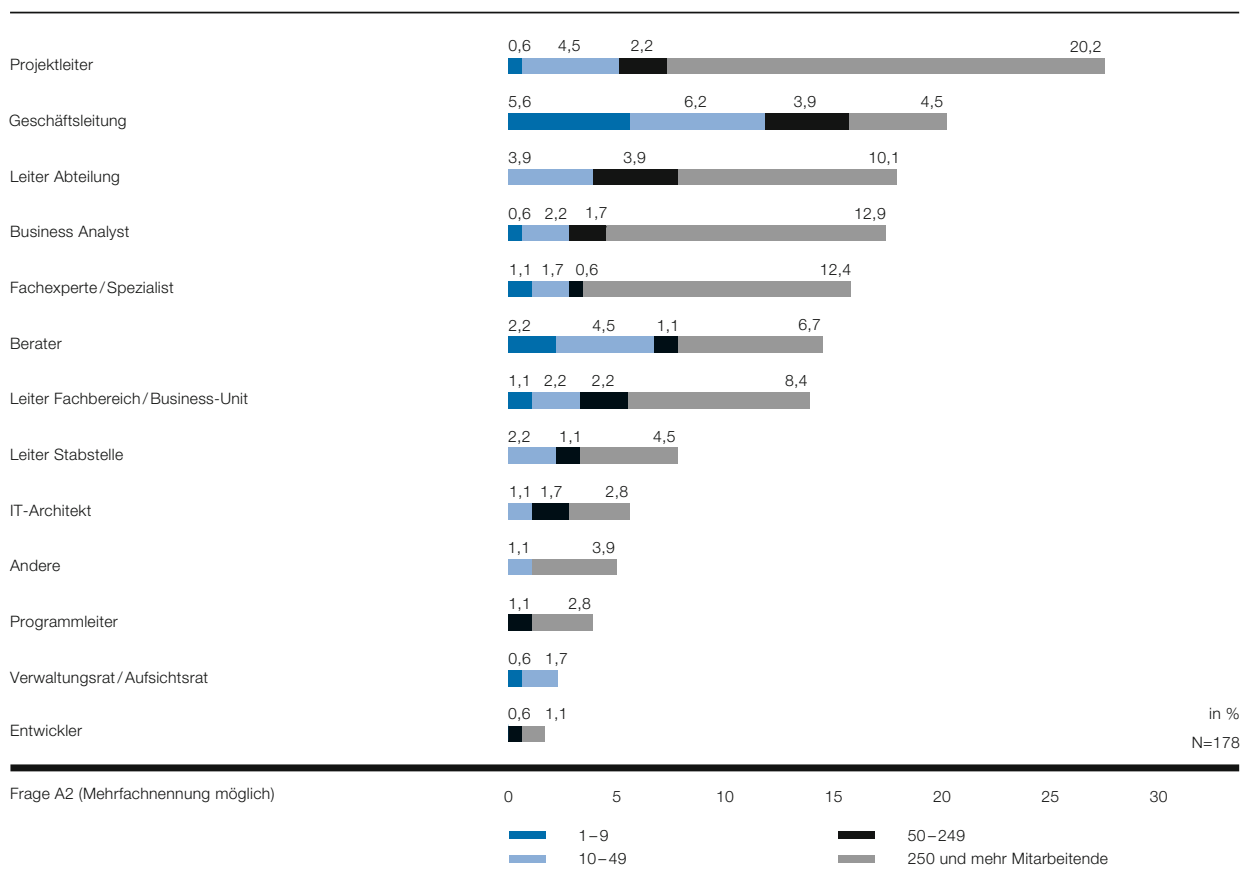

- Abb. 10.5 Teilnehmende nach Position

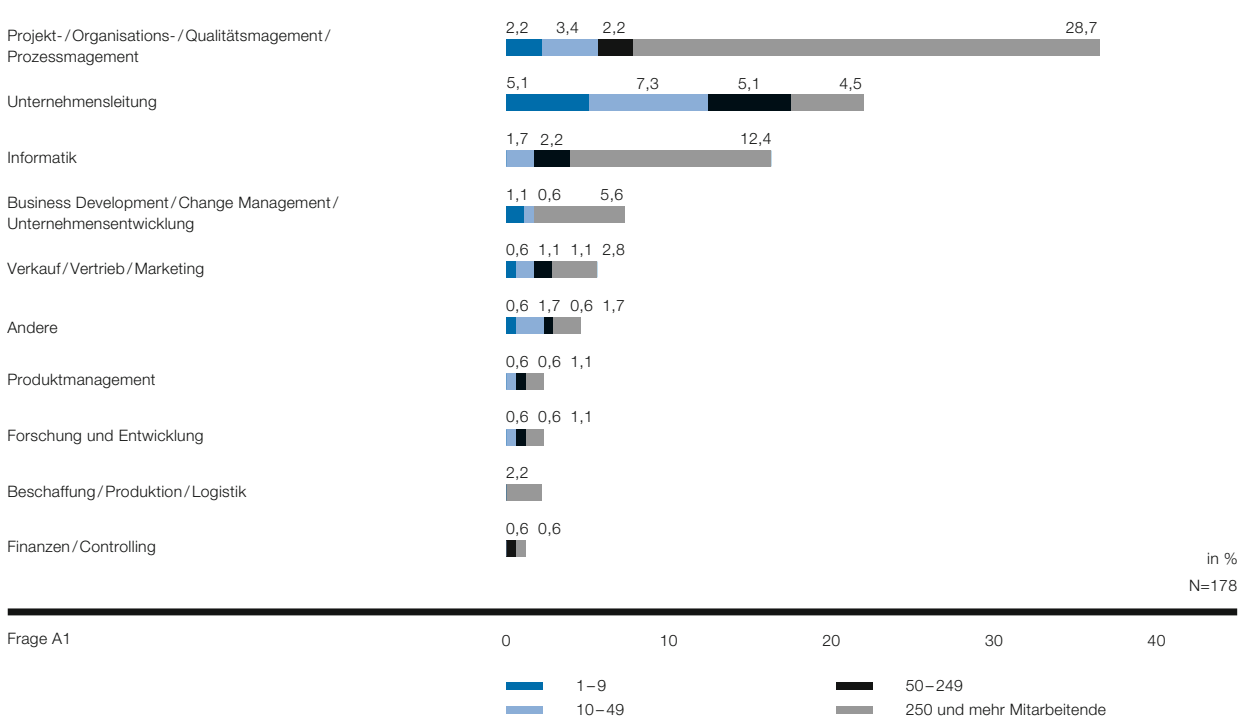

- Abb. 10.6 Teilnehmende nach Funktionsbereich 


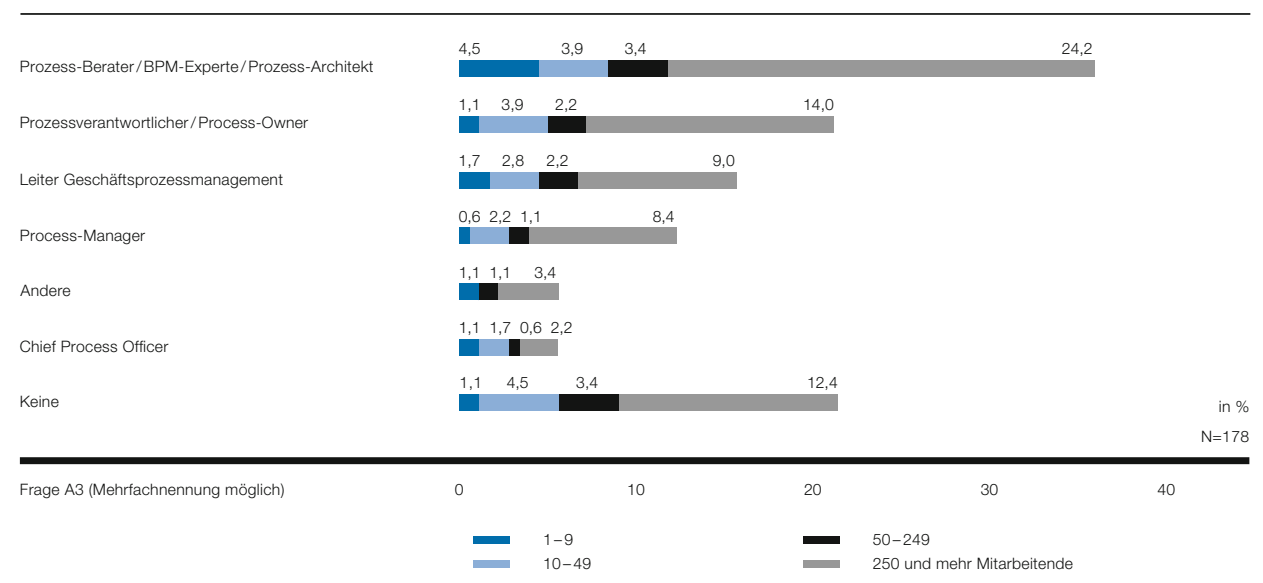

- Abb. 10.7 Teilnehmende nach Wahrnehmung einer Prozessfunktion 


\subsection{Fragenkatalog der Online-Befragung}

Fragen und Antwortmöglichkeiten

Antworttyp

\section{A - Allgemeine Fragen}

A1

\section{In welchem Funktionsbereich Ihres Unternehmens sind Sie tätig?}

Single

Unternehmensleitung

Forschung und Entwicklung

Beschaffung/Produktion/Logistik

Verkauf/Vertrieb/Marketing

Business Development/Change Manage-

ment/Unternehmensentwicklung

Projekt-/Organisations-/Qualitätsmanagement/Prozessmanagement

Produktmanagement

Finanzen/Controlling

Personalwesen

Informatik

Anderes-Bitte nennen:

Verwaltungsrat/Aufsichtsrat

Geschäftsleitung

Leiter Fachbereich/Business-Unit

Leiter Stabstelle

Leiter Abteilung

Business Analyst

Entwickler

Projektleiter

Programmleiter

Berater

IT-Architekt

Fachexperte/Spezialist

Anderes-Bitte nennen: 


\begin{tabular}{|c|c|c|}
\hline & Fragen und Antwortmöglichkeiten & Antworttyp \\
\hline \multirow[t]{8}{*}{ A3 } & Üben Sie eine Prozessfunktion aus? & Mehrfach \\
\hline & Keine & \\
\hline & Chief Process Officer & \\
\hline & Leiter Geschäftsprozessmanagement & \\
\hline & Prozess-Berater/BPM-Experte/Prozess-Architekt & \\
\hline & Prozessverantwortlicher/Process-Owner & \\
\hline & Prozess Manager & \\
\hline & Andere-Bitte nennen: & \\
\hline \multirow[t]{5}{*}{ A4 } & In welchem Land ist Ihre Organisation primär tätig? & Mehrfach \\
\hline & Schweiz & \\
\hline & Deutschland & \\
\hline & Österreich & \\
\hline & Anderes-Bitte nennen: & \\
\hline \multirow[t]{5}{*}{ A5 } & Wie viele Mitarbeitende zählt Ihre Organisation weltweit? & Single \\
\hline & $1-9$ & \\
\hline & $10-49$ & \\
\hline & $50-249$ & \\
\hline & 250 und mehr & \\
\hline \multirow[t]{13}{*}{ A6 } & Welcher Branche gehört Ihre Organisation an? & Single \\
\hline & Energie- und Wasserversorgung & \\
\hline & Verarbeitung, Produktion, Bau & \\
\hline & Chemie, Pharma & \\
\hline & Immobilienwirtschaft & \\
\hline & Handel, Logistik, Telekommunikation & \\
\hline & Öffentliche Dienstleistungen & \\
\hline & Gesundheitswesen & \\
\hline & Beratung, sonstige Dienstleistungen & \\
\hline & Finanz- und Versicherungsdienstleistungen & \\
\hline & Erziehung, Bildung und Forschung & \\
\hline & Informatik & \\
\hline & Andere-Bitte nennen: & \\
\hline
\end{tabular}


Fragen und Antwortmöglichkeiten

Antworttyp

B - BPM-Ziele \& Digitalisierungs-Aktivitätsbereiche

B1 Welche strategischen Ziele treiben ihr Prozessmanagement? Bitte Mehrfach kreuzen Sie die DREI aktuell wichtigsten an.

Transparenz (für z. B. Prozessbewusstsein, Qualitätssicherung, Erfüllung Regulatorischer Vorschriften, Risikomanagement)

Wirtschaftlichkeit (Effizienz, Produktivität, Ertragskraft)

Flexibilität (Personalisierung von Prozessinstanzen, rasche Anpassung von Prozessen an sich ändernde Rahmenbedingungen)

Kundenzufriedenheit/-nutzen

Innovation (Produkte, Dienstleistungen, Geschäftsmodell)

Andere-Bitte nennen:

B1a Bitte priorisieren Sie diese Ziele, indem Sie sie in die richtige Reihenfolge auf der rechten Seite ziehen (höchste Priorität= zuoberst)

Haben Sie Projekte oder Geschäftsfähigkeiten in den folgenden Bereichen der digitalen Transformation?

Einsatz neuer Technologien an der Schnittstelle zu externen Kunden und Endgeräten/Dingen (Social Media, Mobile, Cloud, Internet der Dinge)

Einsatz neuer Verfahren der Datensammlung und Analyse (Big Data, Kundenkontext, Lokalisierung, predictive analytics)

Einsatz neuer Technologien, um Mitarbeitende flexibler/mobiler und effektiver zu unterstützen (digital workplace)

Automatisierung/Digitalisierung durchgängiger Prozesse (Workflow, Prozessintegration)

Digitale Erweiterung bestehender Produkte/Dienstleistungen

Entwicklung neuer digitaler Produkte/Dienstleistungen

Aufbau/Teilnahme an digitalen Plattformen/Netzwerken mit Partnern (zur Realisierung unternehmensübergreifender Prozesse und Angebote)

Sicherstellung von Datenschutz und Datensicherheit im Kontext der Digitalisierung von Prozessen und Kundeninteraktionen

Optimierung der User Experience (Nutzungserlebnis von Websites, Apps und anderen SW-Oberflächen)

Wir haben eine unternehmensweite Strategie und Roadmap für die digitale Transformation

Andere-Bitte nennen: 
Fragen und Antwortmöglichkeiten

Antworttyp

\section{C-Kundenorientierung}

C1 Beschäftigt sich Ihre Organisation mit Themen, die die Interaktionen mit Ihren externen Kunden betreffen?

nie - punktuell/taktisch -

Erweiterung oder Optimierung digitaler Touchpoints/Kanäle

Erweiterung oder Optimierung physischer Touchpoints/Kanäle

(Läden, Verkaufspersonal etc.)

Durchgängige Kundenerfahrung unabhängig vom Touchpoint/Kanal (physisch und digital)

Personalisierte Angebote und Kundeninteraktionen (basierend auf Datenauswertung und Kundenkenntnis)

Flexible, individuell vom Kunden mitgestaltbare Produkte, Dienstleistungen, Interaktionen oder Oberflächen digitaler Touchpoints

Transparenz und Mitbestimmung der Kunden bei der Sammlung, Haltung und Auswertung persönlicher Daten (Personendaten und Interaktions-/Transaktionsdaten)

Flexiblere/kompetentere/schnellere Bedienung des Kunden durch Hilfsmittel in der Hand von Mitarbeitenden mit Kundenkontakt (z. B. Wissensdatenbank auf mobilem Gerät)

Andere-Bitte nennen:

\section{Passen folgende Aussagen auf Ihr Unternehmen?}

Wir sind mit unseren Aktivitäten im Hinblick auf die Kenntnis der Kundenbedürfnisse führend in unserer Branche

Kundenorientierung ist in unserer Marketing-/Unternehmensstrategie verankert

Wir verfügen über die notwendigen Ressourcen (finanziell, personell, Infrastruktur) um die Ausrichtung auf Kundenbedürfnisse voranzutreiben

C3 Was unternehmen Sie, um die Bedürfnisse Ihrer Kunden und Ihrer Mitarbeitenden zu erheben? systematisch/strategisch

nein - teilweise - ja

Wir analysieren die bei uns eingehenden Beschwerden von Kunden

Wir befragen Kunden mit Hilfe quantitativer Methoden (z. B. mit Fragebogen)

Wir befragen Kunden mit Hilfe qualitativer Methoden (z. B. Interviews, Gruppendiskussionen)

Wir prüfen die Kundenakzeptanz von neuen Angebotskonzepten (Prototypen) direkt mit Kunden (z. B. in Produkttests) bevor wir neue Produkte, Dienstleistungen, Interaktionsformen einführen

Wir nutzen die «Customer Journey«, um Bedürfnisse unserer Kunden prozess- und interaktions-orientiert zu erheben

nie - gelegentlich - systematisch/regelmässig 
Fragen und Antwortmöglichkeiten

Wir beobachten Kundenmeinungen in Bezug auf unser Unternehmen im World Wide Web (Social Media Monitoring)

Wir haben Zugriff auf eine Kunden-Community, die bei kundenspezifischen Fragestellungen/Themen aktiv mit uns zusammenarbeitet

Wir nutzen die «Employee Journey«, um die Berührungspunkte der Mitarbeitenden von der Rekrutierung an abzubilden, und die Arbeitsumgebung danach zu gestalten

Wir erheben Kennzahlen, die implizit Rückschlüsse auf die Kundenzufriedenheit bzw. den Kundennutzen zulassen (z. B. Verweilzeit auf der Website, Zuwachs Up- und Cross-selling, Anzahl Service-Anfragen, Anzahl Reklamationen)

Wir sammeln Daten über Einzelkunden und/oder Kundensegmente und werten sie aus

Andere-Bitte nennen:

C3a

Nutzen Sie die Erkenntnisse der «Customer Journey» für die kundenorientierte Gestaltung/Optimierung Ihrer Prozesse?

Ja

Nein

Kann ich nicht beurteilen

C3b Nutzen Sie die Erkenntnisse der «Employee Journey» für die mitarbeiterorientierte Gestaltung/Optimierung Ihrer internen Prozesse und Abläufe (z. B. Rekrutierung, Personalentwicklung)?

Ja

Nein

Kann ich nicht beurteilen

C4 Aus welchen Quellen und wie häufig erheben Sie Kundendaten und Daten, die sie nutzen, um Rückschlüsse auf das Kundenverhalten zu ziehen?

Dediziert erstellte Kundendatenbanken bzw. CRM-Systeme (Customer Relationship Management)

Kern- und Transaktionssysteme

Auswertungen von Kundenumfragen und Kundenfeedbacks/-reklamationen

Soziale Medien (Soziale Netzwerke, Foren, Blogs, Bewertungsplattformen etc.)

Sensoren/Wearables/Dingen (Internet of Things)

Auswertungen von Bewegungs- und Kontextdaten (aus Web-Analysebzw. anderen Tracking-Tools, Lokalisierungsdaten)

Andere-Bitte nennen:
Antworttyp

Single

Single

nie - gelegentlich - systematisch/regelmässig 


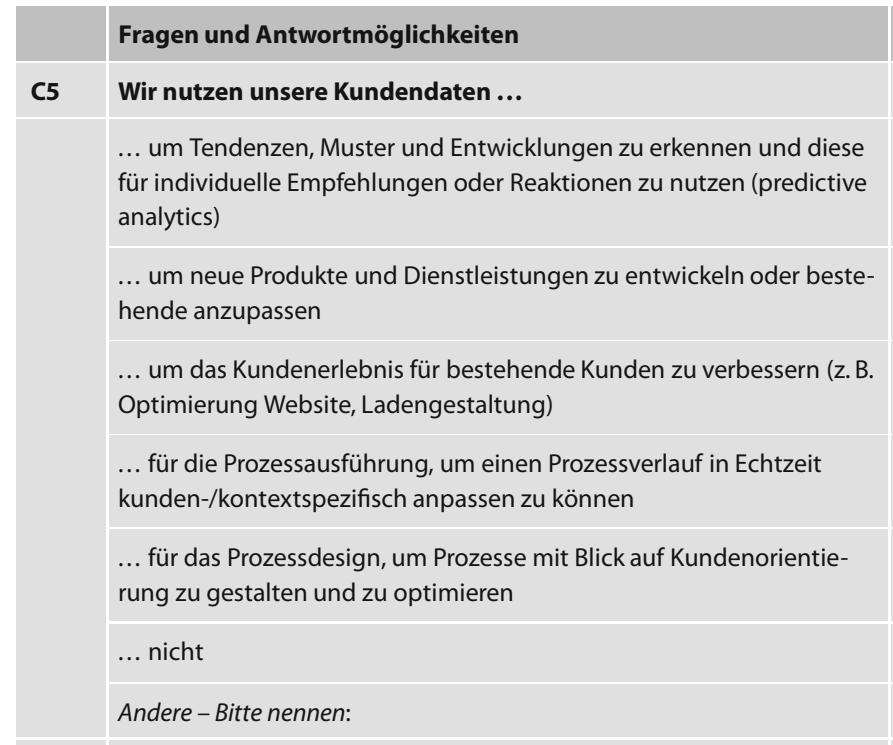

C6 Können ihre Kunden auf die Daten, welche Sie über sie sammeln, Einfluss nehmen? «Unsere Kunden ...
... wissen nicht, welche Daten wir über sie sammeln
... geben uns ihr Einverständnis, bestimmte Daten über sie zu sam- meln

... können veranlassen, dass bestimmte Daten nicht gesammelt oder gelöscht werden

... können bestimmen, ob und wofür wir ihre Daten auswerten

... können bestimmen, welche Informationen sie von uns regelmässig erhalten wollen«

Andere-Bitte nennen:

\section{Antworttyp}

Mehrfach

Mehrfach

\begin{tabular}{|l|}
\hline \\
\hline \\
\hline \\
\hline Mehrach \\
\hline
\end{tabular}


Fragen und Antwortmöglichkeiten

Antworttyp

D - Operational und Service Excellence \& BPM

D1 Gehören folgende Tätigkeiten in Ihrem Unternehmen zum Alltag?

nie - gelegentlich - systematisch/regelmäßig

Drucken von Dokumenten zur Erledigung von Aufgaben

Scannen von Dokumenten

Mehrfacheingaben von gleichen Informationen in unterschiedliche Informatiksysteme

Handschriftliches Unterschreiben von Dokumenten (im Gegensatz zu digitaler Unterschrift)

Individuelle Suche von Informationen (in unterschiedlichen Systemen und Nachfragen bei Personen), um Entscheide treffen zu können

\section{Erheben/Identifizieren Sie folgende entscheidungsunterstützen-} den Informationen?

nie - gelegentlich - systematisch/regelmäßig

Erfolgskritische Prozesse (Wettbewerbsperspektive)

Für die Kundenerfahrung oder -zufriedenheit kritische Prozesse

Standardisierungs- und Automatisierungspotenzial

Digitalisierungspotenziale (über reine Standardisierung/Automatisierung hinaus, z. B. Kollaboration, Kundeninteraktion,

Flexibilisierung etc.)

Potenzial für Prozessintegration über Unternehmensgrenzen hinweg (mit Partnern, Lieferanten)

Out/Sourcing-Potenzial

Optimierungspotenzial der User Experience (Nutzungserlebnis von Websites, Apps und anderen SW-Oberflächen)

Andere-Bitte nennen:

D2a Wo legen Sie den Fokus bei der Optimierung der User Experience (Nutzungserlebnis von Websites, Apps und anderen SW-Oberflächen)?

Auf externe Kunden

Auf Mitarbeitende

Auf Partner/Kooperationen

Andere-Bitte nennen:

D3 Visualisieren Sie Kundeninteraktionen und -bedarf in Ihren Prozessmodellen und -Landkarten?

Nein, wir modellieren weder Prozesslandkarten noch Prozesse

Nein, wir modellieren Prozesse nur aus unserer internen Sicht, ohne die Kunden und die Interaktionen mit Ihnen explizit zu modellieren

Ja, in unseren Prozesslandkarten

Ja, in einzelnen Prozessmodellen 
Fragen und Antwortmöglichkeiten

D4

Wie visualisieren Sie Kundeninteraktionen in Ihren Prozesslandkarten?

Wir visualisieren den Kunden unspezifisch als Block auf der obersten Ebene unserer Prozesslandkarte

Wir visualisieren unterschiedliche Kundengruppen auf unserer Prozesslandkarte

Wir visualisieren unsere Wertschöpfung als End-to-End-Prozesse auf Ebene Prozesslandkarte (konsequent ausgehend vom Kundenbedarf und durchgehend bis zur Leistungserfüllung für den Kunden)

Wir kennzeichnen Prozesse, bei denen wir Kundenkontakt haben und welche für Kundenzufriedenheit/-erfahrung besonders kritisch sind

Andere-Bitte nennen:

D5

Wie visualisieren Sie Kundeninteraktionen in Ihren einzelnen Prozessmodellen?

Wir visualisieren am Prozess beteiligten Kunden mit einem Symbol (Pool, Lane, Swimmlane, ...)

Wir unterscheiden zwischen Kundengruppen durch Nutzung verschiedener Symbole, Farben oder anderer Markierungen

Wir beschriften die Interaktionen mit Detailinformationen oder stellen diese visuell durch Symbole/Farben dar (z. B. Beschriftung der Nachrichtenflüsse mit Kommunikationskanaltyp wie Telefon, E-Mail, Brief, Online etc.)

Wir stellen die mit dem Kunden ausgetauschten Geschäftsobjekte mit einem Symbol dar und geben den angestrebten Zustandsstatus an

Wir kennzeichnen die für Kundenzufriedenheit kritischen Interaktionen speziell

Andere-Bitte nennen:

D6 Welche Methoden setzen Sie ein, um die Flexibilität und Kontextsensitivität Ihrer Prozesse zu ermöglichen?

Keine

Formalisierte Geschäftsregeln (um die Ausführung komplexer Prozesse zu steuern, z. B. durch eine Business Rules Engine)

Formalisierte Ereignisregelung (um auf Ereignisse in der Prozessausführung reagieren zu können, z. B. durch eine Event Engine)

Zugriff auf Wissensbasen (um Entscheide in der Prozessausführung zu unterstützten)

Adaptive Case Management (um Entscheidungen und Prozesssteuerung in wissensintensiven und nur teilweise vordefinierbaren Prozessen zu unterstützen)

Ad-hoc Workflows (um Prozessbeteiligte/Fachexperten bei der Auslösung/Auswahl/Definition von Aktivitäten in nicht vordefinierbaren Prozessen zu unterstützen)

\section{Antworttyp}

Mehrfach

Mehrfach

Mehrfach 


\begin{tabular}{|c|c|c|}
\hline & Fragen und Antwortmöglichkeiten & Antworttyp \\
\hline \multirow{9}{*}{ D7 } & $\begin{array}{l}\text { Agile Methoden (um Fachexperten die kollaborative und intuitive } \\
\text { Modellierung und Anpassung von Prozessen im Rahmen der Pro- } \\
\text { zessausführung zu ermöglichen, z. B. flexibles, beschleunigtes und } \\
\text { praxisnahes Prozess(re)design) }\end{array}$ & \\
\hline & $\begin{array}{l}\text { Prädiktive und selbstlernende Methoden (um auf der Grundlage von } \\
\text { Erfahrungsdaten aus vergangenen Prozessdurchläufen, Rückschlüsse } \\
\text { auf den Ablauf aktueller und künftiger Prozesse zu ziehen, z. B. durch } \\
\text { data mining) }\end{array}$ & \\
\hline & Andere-Bitte nennen: & \\
\hline & $\begin{array}{l}\text { Welche Methoden setzen Sie ein, um die IT-gestützte Ausführung } \\
\text { von Prozessen durchgängig von und zum Kunden zu realisieren? }\end{array}$ & Mehrfach \\
\hline & $\begin{array}{l}\text { Keine, die Ausführung unserer Prozesse ist nicht durchgängig, d. h. } \\
\text { Medienbrüche sind vorhanden }\end{array}$ & \\
\hline & $\begin{array}{l}\text { Spezifische Applikationen/Komponenten/Formulare, die in eine Web- } \\
\text { site oder App integriert sind und die Dateneingaben des Kunden } \\
\text { direkt (ohne Systembrüche) an ein internes System übertragen ( } z \text {. B. } \\
\text { via XML, Webservices) } \rightarrow \text { «Outside-In» }\end{array}$ & \\
\hline & $\begin{array}{l}\text { Anpassung von intern genutzten Applikationen (Oberfläche, Funktio- } \\
\text { nalitäten), so dass sie auch externen Kunden zur Verfügung gestellt } \\
\text { werden können } \rightarrow \text { «Inside-Out» }\end{array}$ & \\
\hline & $\begin{array}{l}\text { Eine BPM/Workflow-Lösung/Plattform/Suite, die Prozesse durchgän- } \\
\text { gig vom Kunden-Frontend (Website, Portal, App) hin zu den Backend- } \\
\text { Systemen und Datenspeichern implementiert bzw. integriert }\end{array}$ & \\
\hline & Andere-Bitte nennen: & \\
\hline
\end{tabular}


Fragen und Antwortmöglichkeiten

Antworttyp

\section{E - Technologie}

E1 Mit welchen Mitteln reagiert lhre Informatik auf die Herausforde- Mehrfach rungen des unternehmerischen und technologischen Wandels?

Gar nicht

Durch Serviceorientierung (SOA)

Durch die Adoption von neuen Produkten bzw. neuen Produktfunktionalitäten

Durch Agile Methoden im Projektmanagement und in der Softwareentwicklung

Durch eine bimodale Architektur, die es erlaubt stabile Kern- und Supportapplikationen auf der einen Seite und agile kurzlebigere Lösungen auf der anderen Seite in zwei verschiedenen Umgebungen zu entwickeln und zu betreiben

Durch die kurzfristige Beschaffung oder Entwicklung von sehr spezifischen best-of-breed Lösungen für einzelne Anwendungsfälle

Durch gezieltes Outsourcing bzw. Anbindung von Drittanbietern (z. B. Cloudlösungen), um einen Anwendungsfall komplett oder teileweise abzudecken

Durch die Unterstützung von mobilen Endgeräten (Apps und/oder mobilfähige Webseiten für Mitarbeitende und/oder Kunden)

Durch den Aufbau/Betrieb von Kundenportalen

Andere-Bitte nennen:

Open Access Dieses Kapitel wird unter der Creative Commons Namensnennung 4.0 International Lizenz (http://creativecommons.org/licenses/by/4.0/deed.de) veröffentlicht, welche die Nutzung, Vervielfältigung, Bearbeitung, Verbreitung und Wiedergabe in jeglichem Medium und Format erlaubt, sofern Sie den/die ursprünglichen Autor(en) und die Quelle ordnungsgemäß nennen, einen Link zur Creative Commons Lizenz beifügen und angeben, ob Änderungen vorgenommen wurden.

Die in diesem Kapitel enthaltenen Bilder und sonstiges Drittmaterial unterliegen ebenfalls der genannten Creative Commons Lizenz, sofern sich aus der Abbildungslegende nichts anderes ergibt. Sofern das betreffende Material nicht unter der genannten Creative Commons Lizenz steht und die betreffende Handlung nicht nach gesetzlichen Vorschriften erlaubt ist, ist für die oben aufgeführten Weiterverwendungen des Materials die Einwilligung des jeweiligen Rechteinhabers einzuholen.

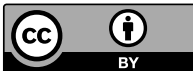

